\title{
A Variational Principle for Black Holes
}

\author{
S. W. Hawking \\ Institute of Astronomy, University of Cambridge, Cambridge, England
}

Received May 20, 1973

\begin{abstract}
It is shown that the initial data which gives rise to stationary black hole solutions extremizes the mass for a given angular momentum and area of the horizon. The only extremum of the mass for a given area of the horizon but arbitrary angular momentum is the Schwarzschild solution. In this case, and when the angular momentum is small, the extremum of the mass is a local minimum. This suggests that the initial data for the Schwarzschild solution has a smaller mass than any other initial data with the same area of the horizon. If this is the case, there is no possibility of proving the occurrence of naked singularities by methods suggested by Penrose and Gibbons. Together with Carter's theorem, the fact that the extremum is a local minimum indicates that the Kerr solutions are stable against axisymmetric perturbations.
\end{abstract}

\section{Introduction}

It has been known for some time that a star of more than about two solar masses can undergo gravitational collapse and produce a black hole. If the collapse is not exactly spherical, the black hole will be initially in an excited state and gravitational radiation will propagate out to infinity and across the event horizon into the black hole. The gravitational radiation reaching infinity will reduce the mass of the system as measured from infinity. $[1,2]$ while the radiation falling into the black hole will cause the area of the event horizon to increase $[3,4]$. If the solution is axisymmetric, the angular momentum will be conserved. Even if the solution is not exactly axisymmetric, it is probable that not all the angular momentum can be radiated away. One would therefore expect the black hole would settle down finally to a stationary equilibrium state which minimized the mass and maximized the area of the event horizon for a given angular momentum. If the black hole is rotating, this stationary state must be axisymmetric [4-6] and be $(t, \phi)$ symmetric, i.e. invariant under $t \rightarrow-t$ and $\phi \rightarrow-\phi[7,8]$. It therefore seems reasonable on physical grounds that among all $(t, \phi)$ symmetric sets of initial data, those which give rise to stationary black hole solutions minimized the mass and maximize the area of the event horizon for a given angular momentum. In fact to determine the position and area of the event horizon, the boundary of the region from which it is not possible to 
escape to infinity, requires a solution of the complete time evolution problem and not simply a knowledge of the initial data. However the initial data is sufficient to determine the location in the initial surface of the apparent horizon $[4,6]$, the outer boundary of the region of the initial surface which contains outer trapped surfaces. An outer trapped surface is a spacelike 2-surface $\mathscr{2}$ such that the outgoing future-directed null geodesics orthogonal to $\mathscr{Z}$ are converging. If the solution is not stationary, the apparent horizon will lie behind the event horizon and its area will be less than that of the event horizon. It therefore follows that one would expect that, among all $(t, \phi)$ symmetric sets of initial data, those sets of initial data which give rise to stationary black hole solutions should minimize the mass and maximize the area of the apparent horizon for a given angular momentum. In this paper I shall show that indeed the mass is extremized for a given area of the apparent horizon and angular momentum. The Schwarzschild solution is the only extremum of the mass for a given area of the apparent horizon but arbitrary angular momentum. At the Schwarzschild solution the mass is a local minimum. This shows that the Schwarzschild solution and spherically symmetrical gravitational collapse are stable against axisymmetric perturbations. It also indicates (modulo certain unproved theorems about function spaces) that the initial data corresponding to the Schwarzschild solution has smaller mass than any other initial data with the same area of apparent horizon. If this is the case, it shows there is no possibility of proving the occurrence of naked singularities by the method proposed by Penrose and elaborated by Gibbons [9]. The idea was to find time-symmetric initial data which had a smaller mass than the Schwarzschild solution with the same area of apparent horizon. Such an initial state could never settle down to a Schwarzschild solution if the area of the horizon increased and the mass decreased.

I shall also show that the extremum of the mass must be a local minimum if the angular momentum is small. Together with Carter's theorem [8] this shows that the Kerr solutions are stable to axisymmetric perturbations.

\section{The Initial Data}

A set of initial data for the empty space Einstein equations consists of a 3-dimensional manifold $\mathscr{S}$ on which there are two symmetric tensor fields $h_{a b}$ and $\chi_{a b}[10,6]$. The tensor field $h_{a b}$ is positive-definitive and the two fields must obey the constraint equations

$$
\begin{gathered}
\chi_{a b \| c} h^{b c}-\chi_{b c \| a} h^{b c}=0, \\
R^{*}+\left(\chi_{a b} h^{a b}\right)^{2}-\chi_{a b} \chi_{c d} h^{a c} h^{b d}=0
\end{gathered}
$$


where $h^{a b} h_{b c}=\delta_{c}^{a}$, the double stroke $\|$ denotes covariant differentiation in $\mathscr{S}$ with respect to the metric $h_{a b}, R^{*}$ is the curvature scalar of $h_{a b}$. A development $\left(\mathscr{M}, \theta, g_{a b}\right)$ of the initial data $\left(\mathscr{S}, h_{a b}, \chi_{a b}\right)$ is a 4-dimensional manifold $\mathscr{M}$ on which there is a Lorentz metric $g_{a b}$ which satisfies the empty space Einstein equations and an embedding $\theta: \mathscr{S} \rightarrow \mathscr{M}$ which is such that $\theta(\mathscr{S})$ is a spacelike surface in $\mathscr{M}, h_{a b}$ is the induced metric $\theta^{*}\left(g_{a b}\right)$ or 1 st fundamental form of $\mathscr{S}$ and $\chi_{a b}$ is the 2 nd fundamental form, $\theta^{*}\left(n_{a ; b}\right)$, where $n^{a}$ is the unit timelike vector orthogonal to $\theta(\mathscr{S})$ in $\mathscr{M}$. The initial data is axisymmetric if it is invariant under, i.e. has zero Lie derivative with respect to, a vector field $\tilde{k}^{a}$ in $\mathscr{S}$ whose orbits are closed curves which vanish on a 1 -dimensional axis. The development of an axisymmetric set of initial data will also be axisymmetric, that is there will be a Killing vector field $\tilde{K}^{a}$ in $\mathscr{M}$ which coincides with $\theta_{*}\left(\tilde{k}^{a}\right)$ on $\theta(\mathscr{S})$. In such an axisymmetric solution one can define the angular momentum $L$ as

$$
-\frac{1}{8 \pi} \int_{2} \tilde{K}_{a ; b} d \Sigma^{a b}
$$

where $d \Sigma^{a b}$ is the surface element of a 2 -surface $\mathscr{2}$ in $\mathscr{M}$. The angular momentum is the same for all homologous 2 -surfaces 2 . It can be evaluated directly from the initial data: $L=+\frac{1}{8 \pi} \int_{\mathscr{P}} \chi_{a b} \tilde{k}^{a} d \Sigma^{b}$ where $d \Sigma^{b}$ is the surface element of a 2 -surface $\mathscr{P}$ in $\mathscr{S}$.

The initial data is said to be $(t, \phi)$ symmetric if it is axisymmetric, if the vector $\tilde{k}^{a}$ is orthogonal with respect to $h_{a b}$ to a family of 2 -surfaces in $\mathscr{S}$ and if there is an isometry of the development which maps $\theta(\mathscr{S})$ into itself but reverses the direction of $n^{a}$ and $\tilde{K}^{a}$ on $\theta(\mathscr{S})$. The necessary and sufficient condition for an axisymmetric set of initial data to be $(t, \phi)$ symmetric is that $\chi_{a b}$ should be of the form

$$
\chi_{a b}=2 J_{(a} \tilde{k}_{b)}
$$

where $J^{a}$ is an axisymmetric vector field orthogonal to $\tilde{k}^{a}$. The angular momentum can then be expressed as

$$
L=\frac{1}{8 \pi} \int_{\mathscr{P}} J_{a} d \Sigma^{a}\left(\tilde{k}^{b} \tilde{k}_{b}\right) .
$$

The first constraint equation will be satisfied if

$$
\left(\tilde{k}^{b} \tilde{k}_{b} J^{a}\right)_{\| a}=0
$$

and the second constraint equation will be satisfied if

$$
R^{*}=2 J^{a} J_{a}\left(\tilde{k}^{b} \tilde{k}_{b}\right) .
$$


One can solve this equation by a method of Lichnerowicz. Choose a spatial metric $h_{a b}$ and find a $J^{a}$ which satisfies Eq. (4) in this metric. One now defines a conformally related metric $\hat{h}_{a b}=V^{4} h_{a b}$ where $V$ is some positive function on $\mathscr{S}$. In the metric $\hat{h}_{a b}$, Eq. (4) will be satisfied by

$$
\hat{J}_{a}=V^{-6} J_{a} \text {. }
$$

Eq. (5) will be satisfied by the initial data $\left(\hat{h}_{a b}, \hat{J}^{a}\right)$ if $V$ is chosen to satisfy

$$
V R^{*}-8 \nabla^{2} V=2 V^{-7} J_{a} J_{b} h^{a b}\left(\tilde{k}^{c} \tilde{k}^{d} h_{c d}\right)
$$

where $R^{*}$ and $\nabla^{2}$ refer to the metric $h_{a b}$.

The initial data will be asymptotically flat if $h_{a b}=\eta_{a b}+O\left(r^{-2}\right)$ and $J^{a}=O\left(r^{-4}\right)$ where $\eta_{a b}$ is the flat space metric on $\mathscr{S}$. In this case, one can define the total mass of the system as

$$
m=-\frac{1}{2 \pi} \lim _{r_{1} \rightarrow \infty} \int_{r=r_{1}} V V_{\| a} d \Sigma^{a} .
$$

The apparent horizon $\partial \mathscr{T}$ in $\theta(\mathscr{S})$ is defined to be the outer boundary of the region $\mathscr{T}$ of $\theta(\mathscr{S})$ which contains outer trapped surfaces. $\partial \mathscr{T}$ will be a marginally outer trapped surface, i.e. the outgoing null geodesics orthogonal to it will have zero convergence. This implies that

$$
\left(\chi_{a b}+w_{a \| b}\right) m^{a} \bar{m}^{b}=0
$$

where $w^{a}$ is a unit vector orthogonal to $\partial \mathscr{T}$ in $\mathscr{S}$ and $m^{a}$ and $\bar{m}^{a}$ are complex conjugate null vectors lying in $\partial \mathscr{T}$ and satisfying $m_{a} \bar{m}^{a}=1$. Since $J^{a}$ will be orthogonal to $\partial \mathscr{T}$, the first term in (9) will be zero. Therefore $w^{a}{ }_{\| a}=0$ which shows that $\partial \mathscr{T}$ is an extremal surface in $\mathscr{S}$ i.e. the area is unchanged to $1 \mathrm{st}$ order under a small deformation. The condition that it is the outer boundary of the region containing outer trapped surfaces implies that in fact it is a surface of minimal area. Thus its area will be less than or equal to the area of the intersection of the initial surface with the event horizon, the boundary of the region from which it is not possible to escape to infinity. The two horizons will coincide and their areas will be equal if and only if the development of the initial data is stationary.

\section{The Variational Formula}

The mass $m$ defined in the last section depended only on the spatial part of the metric. In a stationary metric, that is one that admits a Killing vector $K^{a}$, one can define another quantity $\tilde{m}$ which depends only on the time-like part of the metric.

$$
\tilde{m}=\frac{1}{4 \pi} \int_{2} K_{a ; b} d \Sigma^{a b}
$$


where the integral is taken over a 2 -surface 2 near infinity. If the metric satisfies the empty space Einstein equations near infinity, $\tilde{m}=m$. It was shown in [11] that in a stationary axisymmetric $(t, \phi)$ symmetric black hole metric

$$
\tilde{m}=\frac{\kappa}{4 \pi} A+2 \Omega_{H} L_{H}+\frac{1}{4 \pi} \int_{\mathscr{S}-\mathscr{B}} R_{a b} K^{a} d \Sigma^{b}
$$

where $\kappa$ is the surface gravity of the black hole $\mathscr{B}$ defined by $l^{a}{ }_{; b} l^{b}=\kappa l^{a}$, $l^{a}=K^{a}+\tilde{K}^{a} \Omega_{H}$ is the tangent vector to the generators of the event horizon, $A$ is the area of the event horizon and $L_{H}=-\frac{1}{8 \pi} \int_{\partial \mathscr{B}} \tilde{K}_{a ; b} d \Sigma^{a b}$ is the angular momentum of the black hole. This formula holds whether or not the metric is a solution of the empty space Einstein equations. In a stationary axisymmetric $(t, \phi)$ symmetric metric which satisfies the empty space constraint equations

one can express $\tilde{m}$ as

$$
\left(R^{a b}-\frac{1}{2} R g^{a b}\right) n_{a}=0,
$$

$$
\tilde{m}=\frac{\kappa}{4 \pi} A+2 \Omega_{H} L_{H}+\frac{1}{8 \pi} \int_{\mathscr{S}-\mathscr{B}} R K^{a} d \Sigma_{a} .
$$

One can use Eq. (13) to derive the difference $\delta \tilde{m}$ between the values of $\tilde{m}$ for two nearby axisymmetric $(t, \phi)$ symmetric metrics which satisfy the empty space constraint equations. The calculation is similar to that given in [11]. The variation in the term involving the scalar curvature $R$ gives

$$
-\frac{1}{8 \pi} \int_{\mathscr{S}-\mathscr{B}}\left\{\left(R_{c d}-\frac{1}{2} g_{c d} R\right) f^{c d}+2 f_{[c ; d]}^{c}\right\} K^{a} d \Sigma_{a}
$$

where $f_{a b}=\delta g_{a b}=-g_{a c} g_{b d} \delta g^{c d}$. But

$$
2 f_{[c ; d]}^{c} K^{a}=2\left(K^{a} f_{c}^{[c ; d]}-K^{d} f_{c}^{[c ; a]}\right) ; d
$$

since $f_{c d ; a} K^{a}+f_{a d} K_{; c}^{a}+f_{a c} K_{; d}^{a}=0$ because the perturbation is stationary. One can therefore transform the last term in (14) into the 2-surface integral

$$
-\frac{1}{4 \pi} \int_{\partial \mathscr{B}+\mathscr{P}}\left(K^{a} f_{c}^{[c ; d]}-K^{d} f_{c}^{[c ; a]}\right) d \Sigma_{a d}
$$

where $\mathscr{P}$ is a 2 -surface in $\mathscr{S}$ near infinity. The integral $\partial \mathscr{B}$ gives $-\frac{\delta \kappa}{4 \pi} A-2 \delta \Omega_{H} L$. The integral over $\mathscr{P}$ gives $\delta \tilde{m}-2 \delta m$. Therefore

$$
\delta m=\frac{\kappa}{8 \pi} \delta A+\Omega_{H} \delta L-\frac{1}{16 \pi} \int_{\mathscr{S}-\mathscr{B}}\left(R_{c d}-\frac{1}{2} R g_{c d}\right) f^{c d} K_{a} d \Sigma^{a} .
$$

This formula holds for all variations through stationary axisymmetric $(t, \phi)$ symmetric metrics which satisfy the empty space constraint equations. 
Suppose $\left(h_{a b}, J_{a}\right)$ is a set of $(t, \phi)$ symmetric data which satisfies the empty space constraint equations and which has a development $\left(\mathscr{M}, \theta, g_{a b}\right)$ which is stationary i.e. which admits a Killing vector $K^{a}$. Let $\left(h_{a b}^{\prime}, J_{a}^{\prime}\right)$ be another set of $(t, \phi)$ symmetric initial data on $\mathscr{S}$ which satisfies the empty space constraint equations and which has the same angular momentum and area of the apparent horizon as $\left(h_{a b}, J_{a}\right)$. By a diffeomorphism of $\mathscr{S}$ one can arrange that the apparent horizon of $\left(h_{a b}^{\prime}, J_{a}^{\prime}\right)$ coincides with that of $\left(h_{a b}, J_{a}\right)$. One can then find a stationary axisymmetric and $(t, \phi)$ symmetric metric $g_{a b}^{\prime}$ on $\mathscr{M}$ which satisfies the empty space constraint equations and which is such that $h_{a b}^{\prime}$ and $\chi_{a b}^{\prime}$ are respectively the first and second fundamental forms of $\theta(\mathscr{S})$. The metric $g_{a b}^{\prime}$ can be defined on $\theta(\mathscr{S})$ as

$$
\theta_{*}\left(h^{\prime a b}\right)-n^{\prime a} n^{\prime b}
$$

where $n^{\prime a}=\alpha K^{a}+\beta \tilde{K}^{a}$ and

$$
2 J_{a}=\alpha\left(\beta \alpha^{-1}\right)_{\| a} .
$$

It can be shown that the apparent horizon must be topologically spherical and that the region outside the apparent horizon must be simply connected [4]. Solutions to Eq. (19) will exist in this region. One can then define $g_{a b}^{\prime}$ at other points of $\mathscr{M}$ by dragging it along by the vector field $K^{a}$. This will give a stationary metric $g_{a b}^{\prime}$ on the part of $\mathscr{M}$ that is outside the event horizon in the metric $g_{a b}$. By continuing the metric $g_{a b}^{\prime}$ across the event horizon one obtains a metric whose event and apparent horizons both coincide with the event horizon in the metric $g_{a b}$ and which, outside the event horizon, is stationary, axisymmetric and $(t, \phi)$ symmetric and satisfies the empty space constrain equations (though not necessarily the full Einstein equations). If the initial data $\left(h^{\prime}{ }_{a b}, J^{\prime}\right)$ is near $\left(h_{a b}, J_{a}\right)$, i.e. of the form $\left(h_{a b}+\delta h_{a b}, J_{a}+\delta J_{a}\right)$, then the metric $g_{a b}^{\prime}$ will be of the form $g_{a b}+\delta g_{a b}$ and one can use Eq. (17) to show that $\delta m$ is zero. This shows that the initial data which gives rise to a stationary black hole solution extremizes the mass among all $(t, \phi)$ symmetric initial data with some angular momentum and area of the apparent horizon. Conversely, the $(t, \phi)$ symmetric data which extremizes the mass for given angular momentum and area of the apparent horizon will give rise to a stationary black hole solution.

\section{An Explicit Form of the Variational Principle}

In order to make the variational principle more explicit it is convenient to introduce a special coordinate system in $\mathscr{S}$. By the conditions that the initial data be $(t, \phi)$ symmetric, the metric $h_{a b}$ is $\mathscr{S}$ is invariant under the generator $\tilde{k}^{a}$ of spatial rotations and $\tilde{k}^{a}$ is orthogonal with respect to $h_{a b}$ to a family of 2 -surfaces in $\mathscr{S}$. The 2 -surfaces can be labelled 
by the coordinate $\phi$ with $\phi=0$ identified with $\phi=2 \pi$. In the 2-surfaces $\phi=0$ one can introduce coordinates $\varrho$ and $z$ such that $\varrho=0$ on the axis, $\varrho^{2}+z^{2}=1$ on the apparent horizon and such that the metric induced by $h_{a b}$ on the 2-surface $\phi=0$ has the conformally flat form $e^{2 \zeta}\left(d \varrho^{2}+d z^{2}\right)$. The coordinates $\varrho, z$ can then be defined on the other surfaces $\phi=$ constant by dragging them along with $\tilde{k}^{a}$. The metric $h_{a b}$ then takes the form

where

$$
e^{2 \zeta}\left(d \varrho^{2}+d z^{2}\right)+\varrho^{2} e^{2 \psi} d \phi^{2}
$$

$$
\varrho^{2} e^{2 \psi}=\tilde{k}^{a} \tilde{k}^{b} h_{a b} .
$$

In order to apply the procedure given in Section 2 for solving the constraint equations, choose an $h_{a b}$ of the form (20) with $\zeta=-\beta$ and. $\psi=\beta$ where $\beta$ goes to zero like $\left(\varrho^{2}+z^{2}\right)^{-1}$ at infinity and like $\varrho^{2}$ on the axis. The first constraint equation will be satisfied if

$$
\nabla\left(\varrho^{2} e^{3 \beta} J_{a}\right)=0
$$

where the symbol $\nabla$ refers to the flat space metric in cylindical coordinates $\varrho, z \phi$. If one defines $\lambda$ as $2 \log V$, Eq. (7) becomes [12]

$$
\nabla^{2} \lambda=-\frac{1}{2} \nabla(\lambda+\beta) . \nabla(\lambda+\beta)-\frac{1}{2} \varrho^{2} e^{-4 \lambda+2 \beta} \boldsymbol{J} \boldsymbol{J}-\nabla(\log \varrho) . \nabla \beta
$$

where $\nabla^{2}$ and $\nabla$ and scalar products are taken in the flat space metric in cyclindrical coordinates. Defining $\tilde{\boldsymbol{J}}$ to be $e^{+3 \beta} \boldsymbol{J}$, one can express Eqs. (22) and (23) in the form

$$
\begin{gathered}
\left.\nabla \cdot \varrho^{2} \tilde{\boldsymbol{J}}\right)=0 \\
\nabla^{2} \lambda=-\frac{1}{2} \nabla \mu . \nabla \mu-\frac{1}{2} \varrho^{2} e^{-4 \mu} \tilde{\boldsymbol{J}} \tilde{\boldsymbol{J}}-\nabla(\log \varrho) . \nabla(\mu-\lambda)
\end{gathered}
$$

where $\mu=\lambda+\beta$. Eq. (25) has to be solved with the boundary conditions

where

$$
\lambda=\mu+O\left(r^{-2}\right) \text { as } r \rightarrow \infty
$$

Condition (28) expresses the fact that the sphere $r=1$ is the apparent horizon in the unperturbed metric $h_{a b}$. In performing the variations there is a sufficient freedom in the choice of coordinates that one can keep the apparent horizon at $r=1$. In this case

$$
\frac{\partial(\delta \lambda)}{\partial r}=0 \quad \text { when } \quad r=1 .
$$


I shall impose a different condition to (29) in the following section but in this section it is more convenient to consider unrestricted variations.

In this coordinate system the mass and angular momentum can be expressed as

$$
\begin{aligned}
& m=-\frac{1}{4 \pi} \int_{\mathscr{P}} e^{\lambda} \nabla \lambda \cdot d \Sigma, \\
& L=-\frac{1}{8 \pi} \int_{\partial \mathscr{T}} \varrho^{2} \tilde{\boldsymbol{J}} \cdot d \Sigma .
\end{aligned}
$$

To perform the variation of $m$ subject to the constraints (24) and (25) one constructs the quantity

$$
\begin{aligned}
I= & m-\frac{\kappa}{8 \pi} A-\Omega_{H} L+\frac{1}{8 \pi} \int_{\mathscr{S}-\mathscr{T}} \Omega \nabla\left(\varrho^{2} \tilde{\boldsymbol{J}}\right) \varrho d \varrho d z \\
& +\int_{\mathscr{S}-\mathscr{T}} \xi\left(\nabla^{2} \lambda+\frac{1}{2}(\nabla \mu)^{2}+\frac{1}{2} \varrho^{2} e^{-4 \mu} \tilde{\boldsymbol{J}}^{2}+\nabla(\log \varrho) . \nabla(\mu-\lambda)\right) \varrho d \varrho d z .
\end{aligned}
$$

The stationary black hole solutions will be given by those initial data for which I is an extremum under variations in which $\kappa, \Omega_{H}, \Omega$, and $\xi$ are held constant and $\tilde{\boldsymbol{J}}, \lambda$, and $\mu$ are varied subject to (26) and (27). In performing this variation, $\delta m$ and $\delta L$ are calculated from Eqs. (30) and (31). In the unperturbed metric the apparent horizon $\partial \mathscr{T}$ is at $r=1$ and has area $A=2 \pi \int_{0}^{\pi} e^{2 \lambda} \sin \theta d \theta$ where $\tan \theta=\left(\frac{\varrho}{z}\right)$. In the varied metric the apparent horizon will in general not be at $r=1$ but since the apparent horizon is a minimal surface, its movement does not alter its area to first order. Thus $\delta A=2 \pi \int_{0}^{\pi} e^{2 \lambda} 2 \delta \lambda \sin \theta d \theta$.

The variation with respect to $\lambda$ gives the equations

$$
\begin{array}{r}
\nabla(\varrho \nabla \xi)=0, \\
\xi=0 \text { when } \quad r=1, \\
\frac{\kappa}{4 \pi} e^{2 \lambda}=\frac{\partial \xi}{\partial r} \quad \text { when } \quad r=1, \\
4 \pi \xi=e^{\lambda} \quad \text { when } \quad r=\infty .
\end{array}
$$

Eq. (33) with the boundary conditions (34) and (36) has a unique solution

$$
4 \pi \xi=e^{\lambda_{0}}\left(1-r^{-2}\right)
$$

where $\lambda_{0}$ is the value of $\lambda$ at $r=\infty$. The variation with respect to $\mu$ gives the equation

$$
\begin{aligned}
\nabla(\xi \nabla \mu)= & -2 \xi \varrho^{2} e^{-4 \mu} \tilde{\boldsymbol{J}}^{2} \\
& -\nabla .(\xi \nabla(\log \varrho)) .
\end{aligned}
$$


Multiplying this equation by $\xi^{-1}$, one sees that at $r=1$,

$$
\frac{\partial \mu}{\partial r}=-1
$$

Together with (28) this implies that

$$
\frac{\partial \beta}{\partial r}=0 .
$$

This in turn implies that the null geodesics orthogonal to the 2-surface $\partial \mathscr{T}$ have zero shear. This is a necessary condition for the solution to be stationary, for in a stationary solution the apparent horizon will coincide with the event horizon which will have zero shear [4-6].

The variation with respect to $\tilde{\boldsymbol{J}}$ gives the equations

$$
\begin{array}{lll}
\frac{1}{8 \pi} \nabla \Omega=\xi e^{-4 \mu} \tilde{\boldsymbol{J}}, \\
\Omega=\Omega_{H} & \text { when } & r=1, \\
\Omega=0 & \text { when } & r=\infty .
\end{array}
$$

Eqs. (24), (25), (38), and (41) form a system for determining $\lambda$, $\mu$, and $\tilde{\boldsymbol{J}}$. If one considers variations in which the area of the apparent horizon is held fixed but the angular momentum is allowed to vary, one obtains an extremum of $m$ only when $\Omega_{H}=0$. In this case $\Omega=L=\tilde{\boldsymbol{J}}=0$ and the unique solution is

$$
\lambda=\mu=2 \log \left(1+\frac{1}{r}\right)+\log \left(\frac{m}{2}\right) .
$$

This gives the Schwarzschild metric. Carter's theorem $[8,13]$ shows that the solutions of Eqs. (24), (25), (38), and (41) are locally unique for a given area of the apparent horizon and a given angular momentum. However it has not been ruled out that there can be two or more disjoint solutions when the angular momentum is non-zero.

Given a set of initial data which extremizes the mass for a given angular momentum and area, one can use Eqs. (18), (19) to relate $n^{a}$ to the Killing vector $K^{a}$ with respect to which the metric is stationary. One can then drag the coordinates $\varrho, z$, and $\phi$ on $\mathscr{S}$ along $K^{a}$ and introduce a time coordinate $t$ such that $K^{a} t_{; a}=1$ and $t=0$ on $\mathscr{S}$. In these coordinates the stationary axisymmetric $(t, \phi)$ symmetric black hole solution which arises from the initial data can be expressed as

$$
\begin{aligned}
d s^{2}= & -16 \pi^{2} \xi^{2} e^{-2 \mu} d t^{2}+e^{4 \lambda-2 \mu}\left(d \varrho^{2}+d z^{2}\right) \\
& +\varrho^{2} e^{2 \mu}(d \phi-\Omega d t)^{2} .
\end{aligned}
$$




\section{The Second Variation}

For initial data which gives rise to a stationary black hole solution, the quantity $I$ is an extremum under all variations of $\lambda$, $\mu$, and $\tilde{\boldsymbol{J}}$. This means that the 2 nd order change $\delta^{2} I$ is a quadratic functional of $\delta \lambda, \delta \mu$, and $\delta \tilde{\boldsymbol{J}}$. Similarly the mass $m$ is an extremum under variations which preserve the constraint Eqs. (24) and (25), and leave the angular momentum and area of the apparent horizon unchanged to 1 st order. It therefore follows that $\delta^{2} m$, the 2 nd order change in $m$ for a given angular momentum and area of the apparent horizon, will be equal to $\delta^{2} I$ for variations which to 1 st order satisfy the constraint equations and leave the angular momentum and area unchanged.

In order to evaluate $\delta^{2} I$ it is necessary to calculate the 2 nd order change $\delta^{2} A$ of the area of the apparent horizon produced by 1 st order variation in $\lambda, \mu$, and $\tilde{\boldsymbol{J}}$. In general the apparent horizon $\partial \mathscr{T}$ will suffer a 1 st order displacement from the surface $r=1$ to a new surface $\partial \mathscr{T}^{\prime}$ by such a variation. This displacement does not affect the area to $1 \mathrm{st}$ order but has to be taken into account in calculating $\delta^{2} A$. For variations in $\lambda, \mu$, and $\tilde{\boldsymbol{J}}$ in which $\delta A$ is zero, one can use the coordinate freedom to make a coordinate transformation which makes $\delta \lambda$ and $\delta^{2} \lambda$ zero on $r=1$. Because the new apparent horizon $\partial \mathscr{T}^{\prime}$ is a minimal surface in the varied metric $h_{a b}^{\prime}$, its area will be less than that of $\partial \mathscr{T}$ in the metric $h_{a b}^{\prime}$. But the area of $\partial \mathscr{T}$ in the metric $h_{a b}^{\prime}$ is equal to the area in the unvaried metric $h_{a b}$ since $\delta \lambda=\delta^{2} \lambda=0$. Thus $\delta^{2} A \leqq 0$. Because $\kappa \geqq 0$ one therefore has

$$
\begin{aligned}
\delta^{2} m \geqq m\left(\delta^{\prime} \lambda_{0}\right)^{2}-\delta \lambda_{0}(2 \pi)^{-1} \int_{r=\infty} e^{\lambda} \nabla(\delta \lambda) \cdot d \Sigma \\
\quad+\int_{\mathscr{S}-\mathscr{T}} \xi\left\{(\nabla(\delta \mu))^{2}+\varrho^{2} e^{-4 \mu}\left[8(\delta \mu)^{2} \tilde{\boldsymbol{J}}^{2}-8(\delta \mu) \tilde{\boldsymbol{J}} \delta \tilde{\boldsymbol{J}}\right.\right. \\
\left.\left.\quad+(\delta \tilde{\boldsymbol{J}})^{2}\right]\right\} \varrho d \varrho d z .
\end{aligned}
$$

Eq. (46) holds for all $\delta \lambda, \delta \mu$, and $\delta \tilde{\boldsymbol{J}}$ which obey the constraint equations to 1 st order and are such that $\delta L=0$ and, on $r=1, \delta \lambda=0$. For such perturbations the change $\delta \lambda_{0}$ in the value of $\lambda$ at infinity is zero. Thus the right hand side of (46) is positive definite apart from the term $-8(\delta \mu) \tilde{J} \delta \tilde{\boldsymbol{J}}$. This term is zero for the Schwarzschild metric in which $\tilde{\boldsymbol{J}}=0$. Thus among $(t, \phi)$ symmetric initial data with a given area of apparent horizon, the Schwarzschild initial data corresponds to a local minimum of the mass. Since the Schwarzschild initial data is the only extremum of the mass for a given area, this indicates, modulo certain unproved global theorems about function spaces, that any $(t, \phi)$ symmetric initial data will have a greater mass than the Schwarzschild initial data with the same area of apparent horizon. 
One can also show that $\delta^{2} m$ is positive definite when $\tilde{\boldsymbol{J}}$ is sufficiently small. As $r \rightarrow \infty, \tilde{\boldsymbol{J}}=0\left(r^{-4}\right)$ and $\mu=\lambda_{0}+O\left(r^{-2}\right)$. Therefore there will be some upper bound $C$ to $e^{-2 \mu} r^{2}|\tilde{\boldsymbol{J}}|$ in $\mathscr{S}-\mathscr{T}$. Then

But

$$
-4 e^{-4 \mu} \varrho^{2} \tilde{\boldsymbol{J}} \delta \tilde{\boldsymbol{J}} \delta \mu \geqq-\frac{1}{2} e^{-4 \mu} \varrho^{2}(\delta \tilde{\boldsymbol{J}})^{2}-8 C^{2}(\delta \mu)^{2} r^{-2} .
$$

$$
\begin{aligned}
& \int_{\mathscr{S}} \xi(\delta \mu)^{2} r^{-2} \varrho d \varrho d z=-\int_{\mathscr{S}-\mathscr{T}} \nabla\left(\xi(\delta \mu)^{2}\right) .(\nabla \log r) \varrho d \varrho d z \\
& \leqq \int_{\mathscr{S}-\mathscr{T}} 2 \xi \delta \mu|\nabla(\delta \mu)| r^{-1} \varrho d \varrho d z \\
& \leqq \frac{1}{2} \int_{\mathscr{S}-\mathscr{T}} \xi(\delta \mu)^{2} r^{-2} \varrho d \varrho d z+2 \int_{\mathscr{S}-\mathscr{T}} \xi(\nabla(\delta \mu))^{2} \varrho d \varrho d z .
\end{aligned}
$$

Therefore

$$
\int_{\mathscr{S}-\mathscr{T}} \xi(\delta \mu)^{2} r^{-2} \varrho d \varrho d z \leqq 4 \int_{\mathscr{S}-\mathscr{T}} \xi(\nabla(\delta \mu))^{2} \varrho d \varrho d z .
$$

Using this in (45) one sees that

$$
\begin{aligned}
\delta^{2} m \geqq & \int_{\mathscr{S}-\mathscr{T}} \xi\left\{\left(1-32 C^{2}\right)(\nabla(\delta \mu))^{2}+\frac{1}{2} e^{-4 \mu} \varrho^{2}(4 \delta \mu \tilde{\boldsymbol{J}}-\delta \tilde{\boldsymbol{J}})^{2}\right\} \\
& \cdot \varrho d \varrho d z .
\end{aligned}
$$

This will be positive definite if $32 C^{2}<1$.

It follows that stationary black hole solutions with sufficiently small $\tilde{\boldsymbol{J}}$ will be stable against axisymmetric perturbations since they will correspond to local minima of the mass for given angular momenta and areas of the apparent horizon. In particular the Kerr solutions will be stable for small values of $a / m$. It then follows from Carter's theorem $[13,8]$ that the Kerr solutions must be stable to axisymmetric perturbations for all values of $a / m$ less than 1 , since if there were a value of $(a / m)$ at which the Kerr solutions ceased to be local minima of the mass for a given angular momentum and area, there would be a 1 st order stationary axisymmetric perturbation of the Kerr solution with that value of $(a / m)$. Such a stationary perturbation is shown not to exist by Carter's theorem. A similar result is reached in [14].

I am very grateful to James Bardeen, Brandon Carter, and Gary Gibbons for a number of useful discussions and suggestions.

\section{References}

1. Bondi, H., van der Burg, M. G. J., Metzner, A.W. K.: Proc. Roy. Soc. A 269, 21 (1962)

2. Penrose, R.: Phys. Rev. Lett. 10, 66-68 (1963)

3. Hawking, S. W., Hartle, J. B.: Commun. math. Phys. 27, 283-290 (1972)

4. Hawking, S. W.: The Event Horizon in Black Holes Ed. DeWitt and DeWitt, New York, Paris, London: Gordon and Breach 1973 
5. Hawking, S. W.: Commun. math. Phys. 25, 152-166 (1972)

6. Hawking, S. W., Ellis, G.F.R.: The Large Scale Structure of Spacetime. London: Cambridge University Press 1973

7. Carter, B.: J. Math. Phys. 10, 70-81 (1969)

8. Carter,B.: The General Theory of Stationary Black Hole States in Black Holes Ed. DeWitt and DeWitt, New York, Paris, London: Gordon and Breach 1973

9. Gibbons, G. W.: Commun. math. Phys. 27, 87 (1972)

10. Choquet-Bruhat, Y., Geroch, R.P.: Commun. math. Phys. 14, 329-335 (1969)

11. Bardeen, J. M., Carter, B., Hawking, S. W.: Commun. math. Phys. 31, 161-170 (1973)

12. Bardeen, J.M.: Ap. J. 162, 64 (1970)

13. Carter, B.: Phys. Rev. Lett. 26, 331-332 (1971)

14. Chandrasekhar, S., Friedman, J.L.: Ap. J. 177, 745 (1972)

S. W. Hawking

Institute of Astronomy

University of Cambridge

Cambridge, England 